\title{
Modeling using clinical examination indicators predicts interstitial lung disease among patients with rheumatoid arthritis
}

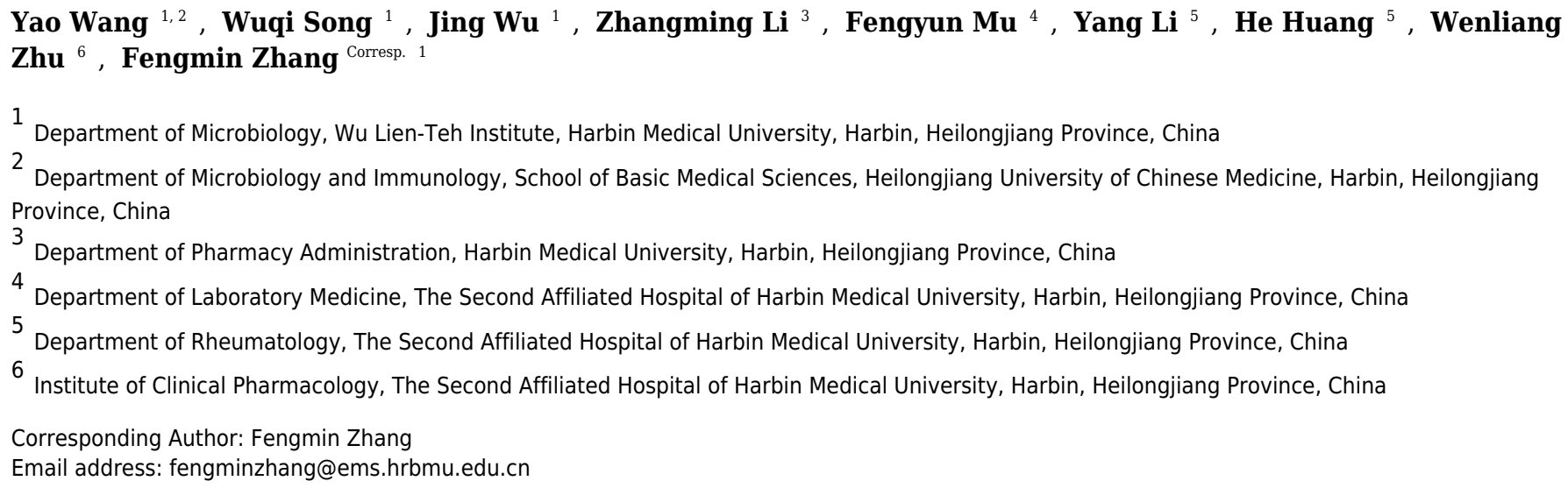

Interstitial lung disease (ILD) is a severe extra-articular manifestation of rheumatoid arthritis (RA) that is well-defined as a chronic systemic autoimmune disease. A proportion of patients with RA-associated ILD (RA-ILD) develop pulmonary fibrosis (PF), resulting in poor prognosis and increased lifetime risk. We investigated whether routine clinical examination indicators (CEIs) could be used to identify RA patients with high PF risk. A total of 533 patients with established RA were recruited in this study for model building and 32 CEls were measured for each of them. To identify PF risk, a new artificial neural network (ANN) was built, in which inputs were generated by calculating Euclidean distance of CEls between patients. Receiver operating characteristic curve analysis indicated that the ANN performed well in predicting the PF risk (Youden index $=0.436$ ) by only incorporating four CEls including age, eosinophil count, platelet count, and white blood cell count. A set of 218 RA patients with healthy lungs or suffering from ILD and a set of 87 RA patients suffering from PF were used for independent validation. Results showed that the model successfully identified ILD and PF with a true positive rate of $84.9 \%$ and $82.8 \%$, respectively. The present study suggests that model integration of multiple routine CEIs contributes to identification of potential PF risk among patients with RA. 
$1 \quad$ Modeling using clinical examination indicators predicts interstitial lung disease among

3 Wang Yao ${ }^{1,2}$, Song Wuqi ${ }^{1}$, Wu Jing ${ }^{1}$, Li Zhangming ${ }^{3}$, Mu Fengyun ${ }^{4}$, Li Yang ${ }^{5}$, Huang He ${ }^{5}$, Zhu

4 Wenliang $^{6}$, Zhang Fengmin ${ }^{1, \#}$

5 'Department of Microbiology, Wu Lien-Teh Institute, Harbin Medical University, Harbin,

6 Heilongjiang Province, China

$7 \quad 2$ Department of Microbiology and Immunology, School of Basic Medical Sciences, Heilongjiang

8 University of Chinese Medicine, Harbin, Heilongjiang Province, China

$9{ }^{3}$ Department of Pharmacy Administration, Harbin Medical University, Harbin, Heilongjiang

10 Province, China

$11{ }^{4}$ Department of Laboratory Medicine, the Second Affiliated Hospital of Harbin Medical

12 University, Harbin, Heilongjiang Province, China

13 Department of Rheumatology, the Second Affiliated Hospital of Harbin Medical University,

14 Harbin, Heilongjiang Province, China

$15{ }^{6}$ Institute of Clinical Pharmacology, the Second Affiliated Hospital of Harbin Medical University,

16 Harbin, Heilongjiang Province, China

17 \#Corresponding author: Zhang Fengmin, Ph.D., 157 Baojian Road, Harbin, Heilongjiang Province,

18 150081, China; Phone/Fax: 86-451-8668-9576, Email: fengminzhang@ems.hrbmu.edu.cn. 


\section{Abstract}

Interstitial lung disease (ILD) is a severe extra-articular manifestation of rheumatoid arthritis (RA)

21 that is well-defined as a chronic systemic autoimmune disease. A proportion of patients with RA-

22 associated ILD (RA-ILD) develop pulmonary fibrosis (PF), resulting in poor prognosis and

23 increased lifetime risk. We investigated whether routine clinical examination indicators (CEIs)

24 could be used to identify RA patients with high PF risk. A total of 533 patients with established

25 RA were recruited in this study for model building and 32 CEIs were measured for each of them.

26 To identify PF risk, a new artificial neural network (ANN) was built, in which inputs were

27 generated by calculating Euclidean distance of CEIs between patients. Receiver operating

28 characteristic curve analysis indicated that the ANN performed well in predicting the PF risk

29 (Youden index $=0.436$ ) by only incorporating four CEIs including age, eosinophil count, platelet

30 count, and white blood cell count. A set of 218 RA patients with healthy lungs or suffering from

31 ILD and a set of 87 RA patients suffering from PF were used for independent validation. Results

32 showed that the model successfully identified ILD and PF with a true positive rate of $84.9 \%$ and $82.8 \%$, respectively. The present study suggests that model integration of multiple routine CEIs

34 contributes to identification of potential PF risk among patients with RA. 


\section{Introduction}

36 Rheumatoid arthritis (RA) is a common chronic systemic autoimmune disorder mainly

37 characterized by joint inflammation. Apart from articular tissue, multiple other tissues and organs

38 may be involved in the pathological process of RA. Indeed, extra-articular manifestations (EAMs)

39 have become the main cause of the morbidity and mortality of patients with RA [1,2]. Among the

40 recognized EAMs, interstitial lung disease (ILD) follows cardiac manifestations [3] as the second

41 contributor to the excess mortality (10\% to $20 \%)$ of patients with RA [4]. Compared with the

42 median survival of 9.9 years for RA alone, patients with RA-associated ILD (RA-ILD) have been

43 reported to have poor prognosis with a median survival of 2.6 years [5]. However, the situation

44 might be worse if pulmonary fibrosis $(\mathrm{PF})$ is also confirmed. In a clinical study focusing on fibrotic

45 interstitial pneumonia, Solomon and colleagues verified that RA patients with fibrotic ILD had

46 worse survival than those with non-fibrotic ILD [6], indicating that PF is an independent risk for

47 mortality in RA.

48 PF causes the aggressive deterioration of lung function and leads to poor prognosis of RA.

49 Unfortunately, fibrotic ILD, especially the subtype usual interstitial pneumonia, still lacks targeted

50 therapy [7]. This leads to worse outcomes in such patients. Nevertheless, early identification of

51 patients with high PF risk would definitely benefit individual RA patient management, in which

52 joint participation of multidisciplinary doctors has been suggested for driving treatment decisions

53 [8]. In the clinical context, any decision making is dependent on diversified clinical examinations

54 including a large number of clinical examination indicators (CEIs). The collection of numerous 
55 CEIs comprehensively reflects the current pathophysiological condition of the patient. In this

56 study, we hypothesized that integration of the CEIs may reveal the potential risk of an individual

57 patient suffering from PF. If the assumption is valid, early risk assessment may be made on

58 admission. To validate the rationality and feasibility of this hypothesis, we performed a

59 retrospective study, in which 620 patients with established RA were included and their clinical

60 examination results were retrieved from the electronic medical records system of the hospital. A

61 novel artificial neural network (ANN) was considered for abstracting and integrating significant

62 information related to PF risk from CEIs. Especially, rather than the traditional ANN and neural

63 network cascade previously described [9-11], the new ANN built here was thought to be a

64 derivative information integration system, in which inputs were generated by calculating the

65 Euclidean distances of CEIs between patients. In conclusion, such an effort aims to provide a

66 viable clinical approach to identify patients with high PF risk and facilitate implementation of early

67 preventive interventions.

68

\section{Methods}

\section{Ethical statement}

71 This study is a retrospective study that was approved by the Ethics Committee of Harbin Medical

72 University (HMU) (Approval number: HMUIRB20150028) and was carried out in accordance

73 with the Declaration of Helsinki.

\section{Patients}


75 The electronic medical record systems of the first and second affiliated hospitals of HMU were

76 used to access the medical records of hospitalized patients that were clinically diagnosed with RA.

77 For each patient, high-resolution computed tomography (HRCT, Discovery CT 750 HD, GE

78 Medical Systems, LLC., Waukesha, WI, USA) was performed to examine whether the

79 complication of ILD or PF was present. In the second affiliated hospital of HMU, 32 CEIs were

80 retrieved from the hospital's electronic system of medical records (form January 1, 2013 to

81 December 31, 2015). All the CEIs were categorized into three classes. The three classes were basic

82 information ( 2 items), routine blood test ( 22 items), and routine urine test ( 8 items). In the first

83 affiliated hospital of HMU, four CEIs including age, eosinophil count (EO), platelet count (PLT),

84 and white blood cell count (WBC) were retrieved from the hospital's electronic system of medical

85 records (from January 1, 2014 to December 31, 2015). If a patient's clinical examination was

86 inadequate, he or she was not taken into consideration for inclusion as a subject. It should be

87 especially noted that there were no other reasons for rejecting subject inclusion, such as age or

88 gender.

89 Identification of ILD-associated CEIs

90 The software MedCalc v15.8 (MedCalc, Mariakerke, Belgium) was used to perform receiver

91 operating characteristic (ROC) curve analysis and calculate the Youden index for each CEI. The

92 Youden index was the sum of sensitivity and specificity minus 1 as defined previously [12]. This

93 effort aimed to investigate whether a CEI can be used as a marker to distinguish patients suffering

94 from ILD from those with healthy lungs. It should be noted that only the RA patients with healthy 
95 lungs and those suffering from ILD were included in the calculation of the Youden index and the

96 subsequent construction of networks and models. ILD-associated CEIs were identified only when

97 the area under the ROC curve (AUC) was significantly larger than $0.5(P<0.05)$, and were retained

98 for further integration by ANN.

99 Data preprocessing and model integration of ILD-associated CEIs

100 ILD-associated CEIs were normalized into a 0 to 1 number before further analysis as previously

101 described [9]. The Intelligent Problem Solver (IPS) tool in the software STATISTICA Neural

102 Networks (SNN, Release 4.0E; Statsoft, Tulsa, OK, USA) was applied to construct a radial basis

103 function (RBF)-ANN model to investigate the effect of CEI integration on ILD association. In this

104 study, the model was simply named as ANN I. The model output then underwent normalization

105 processing and Youden index calculation to investigate whether an obvious association with ILD

106 status was still present after CEI integration. The holdout cross-validation method was applied for

107 preliminary validation of the model as IPS randomly divided the patients into three subsets

108 (training set, verification set, and testing set) in a 2:1:1 ratio. Thus, one-quarter of all the patients

109 did not participate in model building and were used for model testing. The IPS calculated

110 correlation coefficients for the training set $\left(\mathrm{R}_{\mathrm{Tr}}\right)$ and the testing set $\left(\mathrm{R}_{\mathrm{Te}}\right)$. The two correlation

111 coefficients measured the correlation between model output and status of ILD. Similar values of

$112 \mathrm{R}_{\mathrm{Tr}}$ and $\mathrm{R}_{\mathrm{Te}}$ indicates good generalization ability of the model.

113 Euclidean distance calculation and construction of patient-patient similarity network

114 For any two patients, we calculated their Euclidean distance in an $n$-dimensional space, in cases in 
115 which ILD-associated CEIs were re-defined as space coordinates. The value of $n$ was the sum of

116 ILD-associated CEIs. Following the clustering algorithm proposed by Rodriguez and Laio [13],

117 we established a patient-patient similarity network (PPSN) by using the network data visualization

118 software Cytoscape v2.8.3 (Institute of Systems Biology, Seattle, WA, USA) [14].

119 Model integration of derivative information of patients

120 Construction of the ANN I model and the calculation of Euclidean distance of CEIs between

121 patients was used to obtain derivative information for each patient. For example, four items of

122 derivative information could be obtained for patient $i$ as follows: first, we divided the other patients

123 except patient $i$ into $m$ mutually exclusive divisions of nearly equal size according to the magnitude

124 of their distances to patient $i$ in the n-dimensional space of ILD-associated CEIs. Thus, the four

125 items of derivative information might be the ANN I output of patient $i$, mean ANN I output of

126 patients in a given division, mean Euclidean distance to the patients in the division, and actual

127 proportion of RA-ILD among patients in the division. The four derivative information items were

128 imported in a new RBF-ANN as inputs to predict patients' potential PF risk, in the same way as

129 the ANN I. In order to distinguish it from ANN I, the new ANN was named as ANN II. We further

130 investigated the effect of different patient groupings on the performance of ANN II. In this study,

131 the division size $m$ was assigned a value of 5, 10, 15 or 20. For example, if we divided patients

132 into five divisions, we could obtain five candidates of ANN II. The Youden index calculation was

133 then used to identify the best model as ANN II.

134 Model validation and performance evaluation 
135 For ANN II, the 10-fold cross-validation method was used for model validation as previously 136 described [10]. Briefly, all the patients were randomly divided into 10 mutually-exclusive sets of 137 nearly equal size. Next, nine were selected for model training and one was used for model 138 validation. The above procedure was repeated 10 times to allow each of the 10 patient sets to be 139 independently used for validation.

140 To investigate whether the ANN models I and II identify patients with high PF risk, we 141 performed ROC curve analysis on ILD-associated CEIs and outputs of ANN I and ANN II using 142 MedCalc v15.8. Besides AUC, we also recorded the values of sensitivity, specificity, Youden 143 index, and calculated diagnostic odds ratio (DOR) at the optimal cut-off point. The Youden index 144 was the sum of sensitivity and specificity minus 1 as defined previously [12]. According to the 145 definition of DOR in previous studies [15,16], it was calculated as follows:

$$
D O R=\frac{\text { sensitivity } \times \text { specificity }}{(1-\text { sensitivity }) \times(1-\text { specificity })}
$$

152 the discriminant threshold. TPR was calculated as follows:

$$
T P R=\frac{T P}{T P+F N} \times 100 \%
$$


154 where TP and FN are abbreviations of true positive and false negative.

\section{Statistical analysis}

156 MedCalc v15.8 was used to perform pairwise comparisons of the ROC curves based on the 157 methodology of DeLong et al. [17]. Differences were considered as statistically significant when $158 P<0.05$.

159

160

\section{Results}

\section{Patients}

A total of 838 patients with RA were included in this study. Among them, 620 patients from the second affiliated hospital of HMU were subjected to complete clinical examination (Tables S1 and S2). HRCT examination verified the complication of PF in 87 of the 620 patients. In addition, 169

patients were identified as having healthy lungs and 364 were diagnosed as complicated with ILD.

For 218 patients with RA from the first affiliated hospital of HMU, only four CEIs were retrieved identified as having healthy lungs and 146 were diagnosed as complicated with ILD.

\section{Identification of ILD-associated CEIs}

170 For each of the 32 CEIs (Table S1), we investigated any potential association with the status of 171 ILD. Compared with patients with healthy lungs, those RA patients suffering from ILD have an 172 implied greater PF risk [3,4]. Eight CEIs were identified as ILD-associated CEIs (Table 1). For an 173 ILD-associated CEI, higher Youden index suggested better effectiveness as diagnostic marker of 
174 PF risk (Table 1). For instance, among the 32 CEIs, age was assigned the highest Youden index

175 or 0.301 (Figure 1A). This characteristic was highlighted in the pairwise comparisons of the ROC

176 curves as compared with EO, PLT or WBC (the methodology of DeLong et al., P $<0.001$, Figure

177 1B). The Youden index calculation indicated that age and three blood CEIs (EO, PLT, and WBC)

178 were assigned the highest Youden indices, implying relatively closer association with ILD status

179 (Figure 1A). Consistent with this, the optimal ANN model effect was obtained by using the four

180 CEIs (age, EO, PLT, and WBC) as joint inputs of ANN I (Figure S1).

181 Euclidean distance calculation for networking patients' similarity

182 By refining the four ILD-associated CEIs (age, EO, PLT, and WBC) as coordinates in a four-

183 dimensional space, we calculated the Euclidean distance between any two patients and mapped a

184 PPSN for the 533 RA patients including 169 with healthy lungs and 364 complicated with ILD

185 (Figure 2). In spite of the application of different edge settings, a huge patient cluster, rather than

186 multiple scattered patient clusters, was always observed in the network.

\section{Application of ANN for integration of ILD-associated CEIs}

An RBF-ANN with 4-12-1 architecture, named ANN I, was constructed for integration of the four

CEIs that were related to PF status, namely age, EO, PLT, and WBC. Compared with single CEIs, the outputs of ANN I had a better ability to identify patients with high PF risk (Youden index $=$

0.387, Table 2). Furthermore, we built a series of RBF-ANNs with 4-12-1 architecture by

calculating Euclidean distance among patients, distributing patients into divisions, and generating 
194 by the division containing most similar patients showed the best effect in identifying potential PF

195 risks (Figure 3A). Compared with smaller grouping size, the five division method led to creation

196 of an optimal ANN model, namely ANN II (Youden index $=0.436$, Table 2). A significant

197 difference was observed in AUC when ANNs I and II were compared $(P=0.025$, Figure 3B),

198 suggesting the advantage of ANN II in identifying potential PF risk. The DOR of ANN I was 5.41

199 and that of ANN II was 6.88. When specificity was fixed as 0.80 , the sensitivity of ANN I was

2000.473 while that of ANN II was 0.591. These assessments indicated that ANN II was more

201 effective and sensitive than ANN I in identifying patients with high PF risk.

\section{Evaluation of the models}

203 The holdout cross-validation method was used for preliminary validation of the ANNs established

204 in this study. Two model generalization ability parameters $\mathrm{R}_{\mathrm{Tr}}$ and $\mathrm{R}_{\mathrm{Te}}$ were calculated using $\mathrm{SNN}$

205 software. Similar values of $\mathrm{R}_{\mathrm{Tr}}$ and $\mathrm{R}_{\mathrm{Te}}$ indicated that the model built using the IPS tool had good

206 generalization ability. For $A N N$ I, $R_{T r}$ and $R_{T e}$ were 0.398 and 0.440 , respectively, while for ANN

207 II, $\mathrm{R}_{\mathrm{Tr}}$ and $\mathrm{R}_{\mathrm{Te}}$ were 0.461 and 0.489 , respectively. For ANN II in particular, the 10-fold cross-

208 validation method was applied for further model validation (Figure 3C). The AUC was 0.751,

209 implying effectiveness of ANN II in identifying potential PF risk among the patients. Furthermore,

210 an independent set of 87 RA patients complicated with established PF was used to explore whether

211 patients with PF could be identified by single ILD-associated CEIs, ANN I, or ANN II. Compared

212 with single ILD-associated CEIs, ANNs I and II had better recognition for these patients $(\mathrm{TPR}=$

$21382.8 \%$, Figure 4A). Consistent with this, a dot column chart visually showed that patients with 
214 high PF risk and those that had been complicated with PF could be successfully identified with

215 higher sensitivity and specificity using ANN II (Figure 4B). A validation set of patients from the

216 first affiliated hospital of HMU was used for further assessing the model effect of ANN II (Table

217 S3, Figure 4C). The AUC was 0.792, implying the effectiveness of ANN II to identify ILD. A TPR

218 of $84.9 \%$ was calculated for identifying ILD by drawing a $2 \times 2$ contingency table of the validation

219 set (Table S4).

220

\section{Discussion}

Approximately $10 \%$ of patients with RA have ILD-related complications, leading to varying degrees of functional and structural impairment of the lungs [18-20]. This demands clinical management targeted to those patients with RA-associated ILD, especially those with RAassociated fibrotic-ILD [21,22]. For this purpose, it is important to develop early identification of patients with high risk of pulmonary complications $[23,24]$. In the present study, a global analysis of 32 CEIs was performed to reveal clinical predictors that related to high risk of fibrotic-ILD. A modified ANN model was built for CEI integration and detection of risk of fibrotic-ILD among patients with RA.

In this study, the Youden index, instead of Spearman's rho, was applied to explore potential associations between ILD status and CEIs without any ANN data transformation. The choice of Youden index for screening disease-associated CEIs was based on the consideration that the presence or absence of ILD belonged to a logical variable rather than a continuous variable. By 
234 calculating the Youden index, four of the 32 CEIs (age, EO, PLT, and WBC) were identified as

235 having a relatively closer association with ILD status. This result implies multifactorial

236 involvement of pulmonary complications in RA. Among the four ILD-associated CEIs, age

237 showed the strongest association with ILD status (Youden index $=0.301$ ). Older age might lead

238 to a greater risk of fibrotic ILD. This result was in line with a previous study performed by

239 Yilmazer and colleagues [25]. Their study suggested that age was an independent risk factor for

240 lung damage caused by RA-associated ILD. Obvious immunity system alterations and high

241 infection rate were observed in RA patients with ILD [26,27]. In the present study, EO, PLT, and

242 WBC were found to be significantly associated with RA-ILD. White blood cells are a large

243 category of immune system cells, which prevent damage to the body caused by foreign invaders

244 and infectious disease. Eosinophils are a type of white blood cell, while it has been traditionally

245 recognized that platelets are a type of blood cell which plays a central role in physiological

246 hemostasis and pathological thrombosis. Just recently, it was discovered that platelets also act as

247 inflammatory effector cells and are importantly implicated in pathological infectious and immune

248 responses of the lungs [28]. Taken together, abnormalities in the count of immune cells suggest

249 that the lungs of patients with RA-ILD might be subject to infection leading to further tissue

250 damage, such as pulmonary fibrosis. Obviously, aging reduces the ability of the immune system

251 to prevent disease [29]. However, further investigation will be necessary.

252 A PPSN was established by refining the four ILD-associated CEIs as coordinates in a four-

253 dimensional space and calculating Euclidean distance between any two patients. The existence of 
254 a huge cluster of similar patients in the network implied a concentrated distribution of the majority

255 of patients based on the results of routine medical tests (Figure 2). Despite this, it was found that

256 subtle differences in CEIs contributed to heterogeneous local clustering of patients with healthy

257 lungs and those complicated with ILD in the PPSN, suggesting a possibility of joint application of

258 the four CEIs to identify risk of PF.

259 ANN is a universal machine learning method nowadays, which has been widely applied in various areas of medicine, such as decision-making for neurosurgery and prostate cancer diagnosis and medical decision-making. In our study, a decision-making system with novel ANN model architecture was developed for the purpose of facilitating identification of high PF risk among patients with RA. Our results confirmed that integrated processing of ILD-associated CEIs by the derivative information integration system developed here more effectively identified RA patients with high risk of PF, compared with data processing the same CEIs using a traditional ANN (Table requiring multiple operations, including ANN-based CEI integration, establishment of a Euclidean distance-based PPSN, and patient derivative information extraction and integration using an ANN.

270 Although more complex, it was thought that the system would be feasible for clinical practice,

271 because only four routine CEIs were required as network inputs, the model architectures of ANNs

272 I and II were simple, and the Euclidean distance calculation was easy to perform by computer 273 programming. 
274 In conclusion, our study for the first time investigated associations between routine CEIs and ILD

275 in patients with RA using a modified ANN system. The results contributed to new knowledge

276 regarding the identification of patients with high PF risk when routine CEIs were used. Integration

277 of CEIs in a mathematical model facilitated their application in clinical management of such

278 patients. Superior to the traditional ANN model, the developed system consisting of ANNs I and

279 II successfully identified patients at high risk of PF and those having PF among patients with RA

280 by co-considering meaningful associations between CEIs and ILD and similar patients in medical

281 testing. However, two limitations should be noted: our findings were obtained from a small

282 collection of RA patients and only 32 routine CEIs were investigated for their association with

283 ILD. Further research on a larger patient set is definitely needed to validate our results and more

284 CEIs should be considered for investigation.

\section{References}

1. Turesson C. Extra-articular rheumatoid arthritis. Curr Opin Rheumatol. 2013; 25(3):360-366.

2. Sihvonen S, Korpela M, Laippala P, Mustonen J, Pasternack A. Death rates and causes of death in patients with rheumatoid arthritis: a population-based study. Scand J Rheumatol 2004; 33(4):221-227.

3. Brown KK. Rheumatoid lung disease. Proc Am Thorac Soc. 2007; 4(5):443-448.

4. Ingegnoli F, Lubatti C, Ingegnoli A, Boracchi P, Zeni S, Meroni PL. Interstitial lung disease outcomes by high-resolution computed tomograpthy (HRCT) in anti-Jo1 antibody-positive 
294 polymyositis patients: a single centre study and review of the literature. Autoimmun Rev 2012;

$295 \quad 11(5): 335-340$.

296 5. Bongartz T, Nannini C, Medina-Velasquez YF, Achenbach SJ, Crowson CS, Ryu JH, Vassallo

297 R, Gabriel SE, Matteson EL. Incidence and mortality of interstitial lung disease in rheumatoid 298 arthritis: a population based study. Arthritis Rheum 2010; 62(6):1583-1591.

299 6. Solomon JJ, Ryu JH, Tazelaar HD, Myers JL, Tuder R, Cool CD, Curran-Everett D, Fischer 300 A, Swigris JJ, Brown KK. Fibrosing interstitial pneumonia predicts survival in patients with 301 302

7. Travis WD, Hunninghake G, King TE Jr, Lynch DA, Colby TV, Galvin JR, Brown KK, Chung rheumatoid arthritis-associated interstitial lung disease (RA-ILD). Respir Med. 2013; 107(8):1247-1252. doi: 10.1016/j.rmed.2013.05.002. MP, Cordier JF, du Bois RM, Flaherty KR, Franks TJ, Hansell DM, Hartman TE, Kazerooni EA, Kim DS, Kitaichi M, Koyama T, Martinez FJ, Nagai S, Midthun DE, Müller NL,

8. Lake F, Proudman S. Rheumatoid arthritis and lung disease: from mechanisms to a practical approach. Semin Respir Crit Care Med. 2014; 35(2):222-238. doi: 10.1055/s-0034-1371542.

9. Zhu W, Kan X. Neural network cascade optimizes microRNA biomarker selection for nasopharyngeal cancer prognosis. PLoS One. 2014; 9(10):e110537. doi: 10.1371/journal.pone.0110537. 
314 10. Li Z, Li Y, Sun L, Tang Y, Liu L, Zhu W. Artificial neural network cascade identifies multi315 P450 inhibitors in natural compounds. PeerJ. 2015; 3:e1524. doi: 10.7717/peerj.1524.

316 11. Hou S, Wang J, Li Z, Wang Y, Wang Y, Yang S, Xu J, Zhu W. Five-descriptor model to 317 predict the chromatographic sequence of natural compounds. J Sep Sci. 2016; 39(5):864-872. 318 doi: $10.1002 /$ jssc. 201501016.

319 12. Youden WJ. Index for rating diagnostic tests. Cancer. 1950; 3:32-35. doi: 10.1002/10970142(1950)3:1<32::AID-CNCR2820030106>3.0.CO; 2-3.

13. Rodriguez A, Laio A. Machine learning. Clustering by fast search and find of density peaks. Science. 2014; 344(6191):1492-1496. doi: 10.1126/science.1242072.

14. Smoot ME, Ono K, Ruscheinski J, Wang PL, Ideker T. Cytoscape 2.8: new features for data integration and network visualization. Bioinformatics. 2011; 27(3):431-432. doi: 10.1093/bioinformatics/btq675.

15. Böhning D, Holling H, Patilea V. A limitation of the diagnostic-odds ratio in determining an optimal cut-off value for a continuous diagnostic test. Stat Methods Med Res. 2011; 20:541550. doi: 10.1177/0962280210374532.

16. Glas AS, Lijmer JG, Prins MH, Bonsel GJ, Bossuyt PM. The diagnostic odds ratio: a single indicator of test performance. J Clin Epidemiol. 2003; 56:1129-1135. doi: 10.1016/S08954356(03)00177-X.

17. DeLong ER, DeLong DM, Clarke-Pearson DL. 1988. Comparing the areas under two or more correlated receiver operating characteristic curves: a nonparametric approach. 
334

335

336

337

338

339

340

341

342

343

344

345

346

347

348

349

350

351

352

353

Biometrics,1998; 44:837-845.

18. Olson AL, Swigris JJ, Sprunger DB, Fischer A, Fernandez-Perez ER, Solomon J, Murphy J, Cohen M, Raghu G, Brown KK. Rheumatoid arthritis-interstitial lung disease-associated mortality. Am J Respir Crit Care Med. 2011;183(3):372-8. doi: 10.1164/rccm.2010040622OC.

19. Zou YQ, Li YS, Ding XN, Ying ZH. The clinical significance of HRCT in evaluation of patients with rheumatoid arthritis-associated interstitial lung disease: a report from China. Rheumatol Int. 2012;32(3):669-673. doi: 10.1007/s00296-010-1665-1.

20. Richman NC, Yazdany J, Graf J, Chernitskiy V, Imboden JB. Extraarticular manifestations of rheumatoid arthritis in a multiethnic cohort of predominantly Hispanic and Asian patients. Medicine (Baltimore). 2013;92(2):92-97. doi: 10.1097/MD.0b013e318289ce01.

21. Wells AU, Denton CP. Interstitial lung disease in connective tissue disease--mechanisms and management. Nat Rev Rheumatol. 2014;10(12):728-739. doi: 10.1038/nrrheum.2014.149.

22. Mori S. Management of rheumatoid arthritis patients with interstitial lung disease: safety of biological antirheumatic drugs and assessment of pulmonary fibrosis. Clin Med Insights Circ Respir Pulm Med. 2015;9(Suppl 1):41-49. doi: 10.4137/CCRPM.S23288.

23. Moua T, Zamora Martinez AC, Baqir M, Vassallo R, Limper AH, Ryu JH. Predictors of diagnosis and survival in idiopathic pulmonary fibrosis and connective tissue disease-related usual interstitial pneumonia. Respir Res. 2014;15:154. doi: 10.1186/s12931-014-0154-6.

24. Giles JT, Danoff SK, Sokolove J, Wagner CA, Winchester R, Pappas DA, Siegelman S, 
354

355

356

357

358

359

360

361

362

363

364

365

366

367

368

369

370

371

372

373

Connors G, Robinson WH, Bathon JM. Association of fine specificity and repertoire expansion of anticitrullinated peptide antibodies with rheumatoid arthritis associated interstitial lung disease. Ann Rheum Dis. 2014;73(8):1487-1494. doi: 10.1136/annrheumdis-2012-203160.

25. Yilmazer B, Gümüştaş S, Coşan F, İnan N, Ensaroğlu F, Erbağ G, Yıldız F, Çefle A.Highresolution computed tomography and rheumatoid arthritis: semi-quantitative evaluation of lung damage and its correlation with clinical and functional abnormalities. Radiol Med. 2016;121(3):181-9. doi: 10.1007/s11547-015-0590-5.

26. Papanikolaou IC, Boki KA, Giamarellos-Bourboulis EJ, Kotsaki A, Kagouridis K, Karagiannidis N, Polychronopoulos VS. Innate immunity alterations in idiopathic interstitial pneumonias and rheumatoid arthritis-associated interstitial lung diseases. Immunol Lett. 2015;163(2):179-86. doi: 10.1016/j.imlet.2014.12.004.

27. Zamora-Legoff JA, Krause ML, Crowson CS, Ryu JH, Matteson EL. Risk of serious infection in patients with rheumatoid arthritis-associated interstitial lung disease. Clin Rheumatol. 2016;35(10):2585-9. doi: 10.1007/s10067-016-3357-z.

28. Middleton EA, Weyrich AS, Zimmerman GA. Platelets in pulmonary immune responses and inflammatory lung diseases. Physiol Rev. 2016;96(4):1211-1259. doi: 10.1152/physrev.00038.2015.

29. Weyand CM, Goronzy JJ. Aging of the immune system. mechanisms and therapeutic targets. Ann Am Thorac Soc. 2016;13(Supplement_5):S422-S428. doi: 10.1513/AnnalsATS.201602095AW. 
374 30. Hu X, Cammann H, Meyer HA, Miller K, Jung K, Stephan C. Artificial neural networks and 375 prostate cancer--tools for diagnosis and management. Nat Rev Urol. 2013;10(3):174-82. doi: 10.1038/nrurol.2013.9.

377

378

379

380

381

382

383

384

385

386

387

388

389

390

391

392

393

31. Sheikhtaheri A, Sadoughi F, Hashemi Dehaghi Z. Developing and using expert systems and neural networks in medicine: a review on benefits and challenges. J Med Syst. 2014;38(9):110. doi: 10.1007/s10916-014-0110-5.

32. Azimi P, Mohammadi HR, Benzel EC, Shahzadi S, Azhari S, Montazeri A. Artificial neural networks in neurosurgery. J Neurol Neurosurg Psychiatry. 2015;86(3):251-256. doi: 10.1136/jnnp-2014-307807.

\section{Figure legends}

Figure 1. ILD-associated CEIs. A. Distribution of the 32 CEIs in Youden index value. The numbers on the columns indicate the number of CEIs. Four CEIs were observed to have a Youden index of more than 0.15. B. ROC curves of the four ILD-associated CEIs age, eosinophil count (EO), blood platelet count (PLT), and white blood cell count (WBC).

Figure 2. PPSNs of different edges. A. PPSN of 1500 edges; B. PPSN of 2000 edges; C. PPSN of 2500 edges; D. PPSN of 3000 edges. Green dots represent patients with healthy lungs and red dots indicate patients complicated with ILD. Edge between two dots that represent patients means a short Euclidean distance between the two patients in a 4-dimensional space, in which the four ILDassociated CEIs were re-defined as coordinates. Although 533 patients were included in the 
394 calculation of Euclidean distance, only the shortest distances could be visualized as edges in PPSN.

395 For each edge setting, the average number of neighbors was also calculated.

396 Figure 3. Optimization and evaluation of ANN II. A. Distribution of the Youden index values of 397 ANN models built by dividing all the patients with healthy lungs and those complicated with ILD 398 into 5, 10, 15, or 20 groups, respectively. The first ANN models of different division sizes 5D1, 399 10D1, 15D1, and 20D1 are highlighted as larger dots. The position of the dotted line is 0.387 , and 400 values of Youden index were calculated using ANN I. B. Comparison of ANNs I and II in ROC 401 curves. A significant difference in AUCs was found $(P=0.025)$. C. The 10 -fold cross-validation $402(10 \mathrm{FCV})$ result of ANN II.

403 Figure 4. Model evaluation using an independent patient set of 87 RA patients with PF and an 404 external validation set of 218 RA patients. A. Comparison of single ILD-associated CEIs, ANNs 405 I and II in identifying patients with PF. B. Scatter plots of ANN II outputs for patients with healthy 406 lungs and those complicated with ILD or PF. The position of the dotted line is 0.622 , the optimal 407 ROC curve cut-off point of ANN II. C. The external validation result of ANN II.

409 Supplementary information

410 Table S1. Clinical examination results for the 620 patients included in this study.

411 Table S2. Distribution of the 32 clinical examination indicators used in this study.

412 Table S3. Clinical examination results for the patients included in the validation set $(\mathrm{n}=218)$.

413 Table S4. ILD-associated CEIs. 
414 Figure S1. Optimization of ANN I inputs. The optimal combination of four CEIs is highlighted 415 with a bigger dot indicating the highest Youden index. EO: eosinophil count; PLT: blood platelet 416 count; WBC: white blood cell count. For optimization of ANN I inputs, each of the eight ILD417 associated CEIs was selected alone as ANN I input and Youden index was calculated for the output 418 of each ANN model. After that, the CEI with the highest Youden index was obtained and the other 419 CEIs were added by the same rule until all of the eight CEIs were simultaneously used as inputs. 


\section{Table $\mathbf{1}$ (on next page)}

Table 1. Comparison of ILD-associated CEls in identifying patients with high PF risk.

CEI: clinical examination indicator; AUC: area under the ROC curve; SE: sensitivity; SP: specificity; $\mathrm{SE}_{\mathrm{SP}=0.8}$ : sensitivity at fixed specificity $=0.8$; DOR: diagnostic odd ratio. $\mathrm{EO}$ : eosinophil count; PLT: blood platelet count; WBC: white blood cell count NEUT: neutrophil count; U-SG: urine specific gravity; U-WBC: white blood cell count in urine; U-WBCH: white blood cell (high power field) in urine. 
1 Table 1. Comparison of ILD-associated CEIs in identifying patients with high PF risk.

\begin{tabular}{|l|l|l|l|l|l|l|}
\hline CEI & AUC & Youden index & SE & SP & SE $=0.8$ & DOR \\
\hline Age & 0.710 & 0.301 & 0.74 & 0.56 & 0.47 & 3.63 \\
\hline EO & 0.562 & 0.164 & 0.64 & 0.53 & 0.25 & 1.96 \\
\hline PLT & 0.569 & 0.165 & 0.55 & 0.62 & 0.26 & 1.95 \\
\hline WBC & 0.587 & 0.173 & 0.45 & 0.73 & 0.32 & 2.15 \\
\hline NEUT & 0.577 & 0.133 & 0.85 & 0.28 & 0.26 & 2.26 \\
\hline U-SG & 0.580 & 0.135 & 0.93 & 0.20 & 0.30 & 3.57 \\
\hline U-WBC & 0.562 & 0.138 & 0.78 & 0.36 & 0.28 & 1.97 \\
\hline U-WBCH & 0.561 & 0.123 & 0.78 & 0.34 & 0.28 & 1.85 \\
\hline
\end{tabular}

2 CEI: clinical examination indicator; AUC: area under the ROC curve; SE: sensitivity; SP: specificity;

$3 \mathrm{SE}_{\mathrm{SP}}=0.8$ : sensitivity at fixed specificity $=0.8$; DOR: diagnostic odd ratio. EO: eosinophil count; PLT:

4 blood platelet count; WBC: white blood cell count NEUT: neutrophil count; U-SG: urine specific

5 gravity; U-WBC: white blood cell count in urine; U-WBCH: white blood cell (high power field) in urine. 


\section{Table 2 (on next page)}

Table 2. Comparison of models in identifying patients with high PF risk.

CEI: clinical examination indicator; AUC: area under the ROC curve; SE: sensitivity; SP: specificity; $\mathrm{SE}_{\mathrm{SP}=0.8}$ : sensitivity at fixed specificity =0.8; $\mathrm{DOR}$ : diagnostic odd ratio; 5D1, 10D1, 15D1, and 20D1 were the first ANN models created by dividing all the patients with healthy lungs and those complicated with ILD into 5, 10, 15, or 20 groups, respectively. 
1 Table 2. Comparison of models in identifying patients with high PF risk.

\begin{tabular}{|l|l|l|l|l|l|l|}
\hline Model & AUC & Youden index & SE & SP & SE $\mathrm{SP}=0.8$ & DOR \\
\hline ANN I & 0.734 & 0.387 & 0.772 & 0.615 & 0.473 & 5.41 \\
\hline $5 \mathrm{D} 1$ & 0.767 & 0.436 & 0.791 & 0.645 & 0.591 & 6.88 \\
\hline $10 \mathrm{D} 1$ & 0.758 & 0.411 & 0.731 & 0.681 & 0.563 & 5.80 \\
\hline $15 \mathrm{D} 1$ & 0.746 & 0.407 & 0.780 & 0.627 & 0.489 & 5.96 \\
\hline $20 \mathrm{D} 1$ & 0.736 & 0.403 & 0.805 & 0.598 & 0.500 & 6.14 \\
\hline
\end{tabular}

2 CEI: clinical examination indicator; AUC: area under the ROC curve; SE: sensitivity; SP: specificity;

$3 \mathrm{SE}_{\mathrm{SP}=0.8}:$ sensitivity at fixed specificity $=0.8$; DOR: diagnostic odd ratio; 5D1, 10D1, 15D1, and 20D1

4 were the first ANN models created by dividing all the patients with healthy lungs and those complicated

5 with ILD into $5,10,15$, or 20 groups, respectively.

6

7

8 
Figure 1

Figure 1. ILD-associated CEls.

A. Distribution of the 32 CEls in Youden index value. The numbers on the columns indicate the number of CEls. Four CEls were observed to have a Youden index of more than 0.15. B. ROC curves of the four ILD-associated CEls age, eosinophil count (EO), blood platelet count (PLT), and white blood cell count (WBC).

A

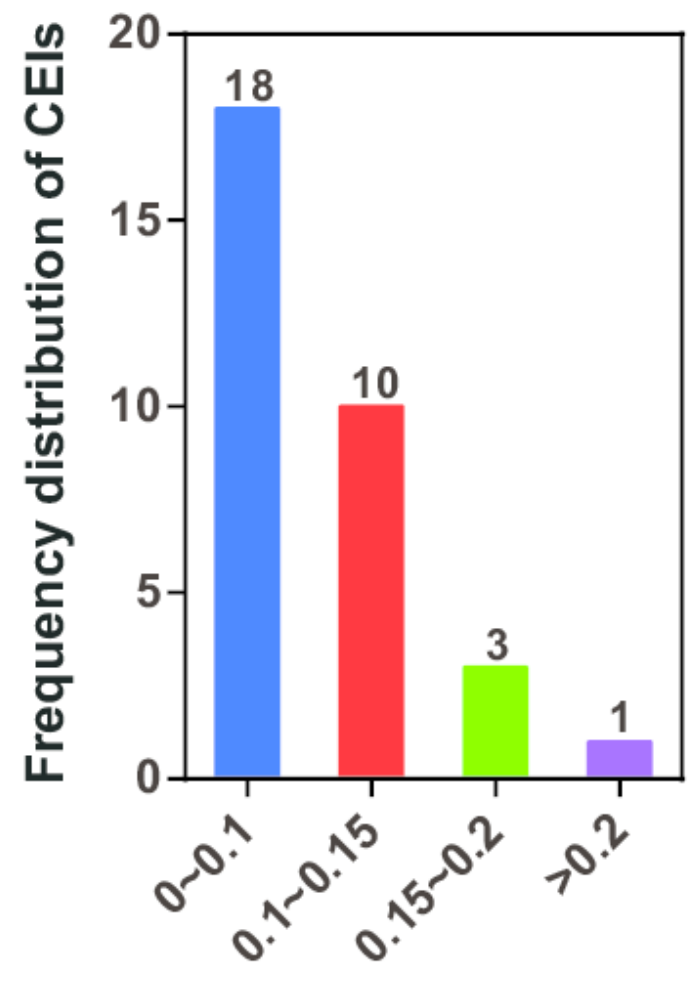

Youden index
B

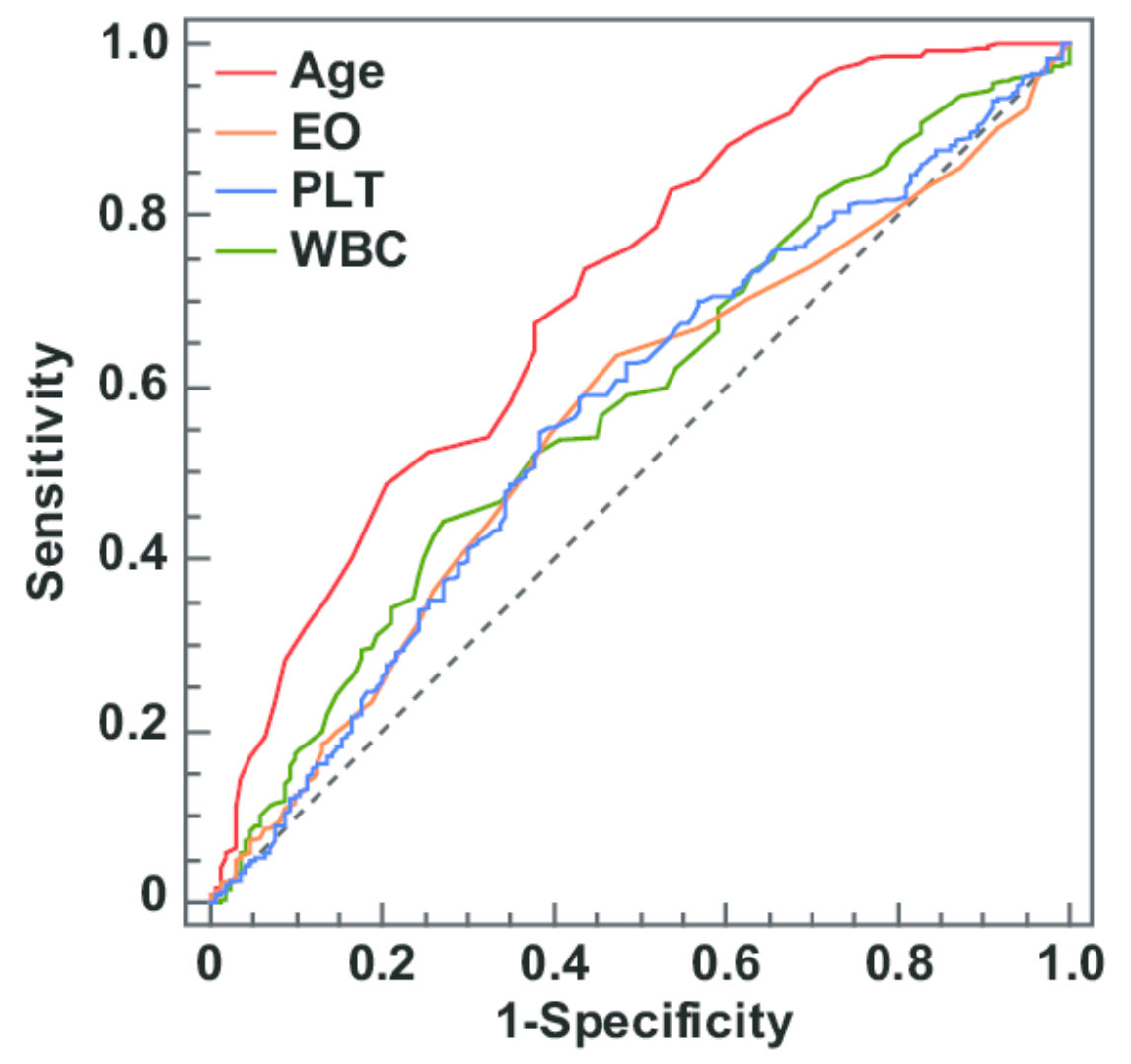




\section{Figure 2}

Figure 2. PPSNs of different edges.

A. PPSN of 1500 edges; B. PPSN of 2000 edges; C. PPSN of 2500 edges; D. PPSN of 3000 edges. Green dots represent patients with healthy lungs and red dots indicate patients complicated with ILD. Edge between two dots that represent patients means a short Euclidean distance between the two patients in a 4-dimensional space, in which the four ILDassociated CEls were re-defined as coordinates. Although 533 patients were included in the calculation of Euclidean distance, only the shortest distances could be visualized as edges in PPSN. For each edge setting, the average number of neighbors was also calculated. 
A

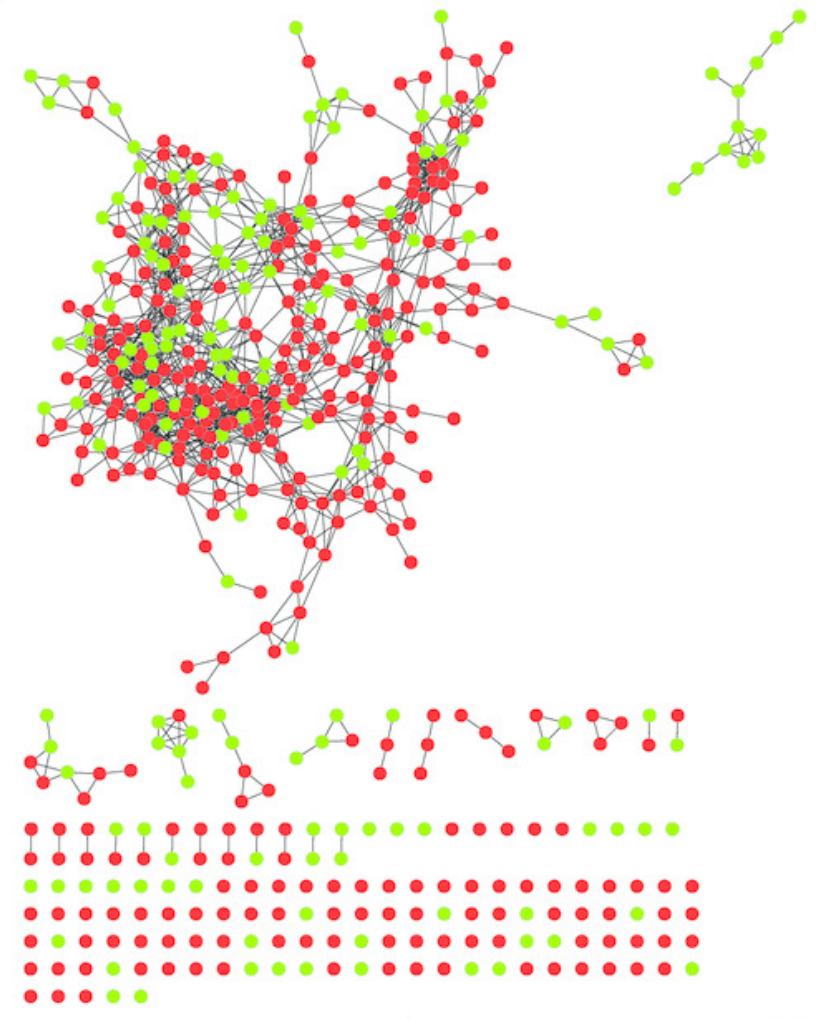

Average number of neighbors $=\mathbf{5 . 6 2 9}$ (1500 edges)

C

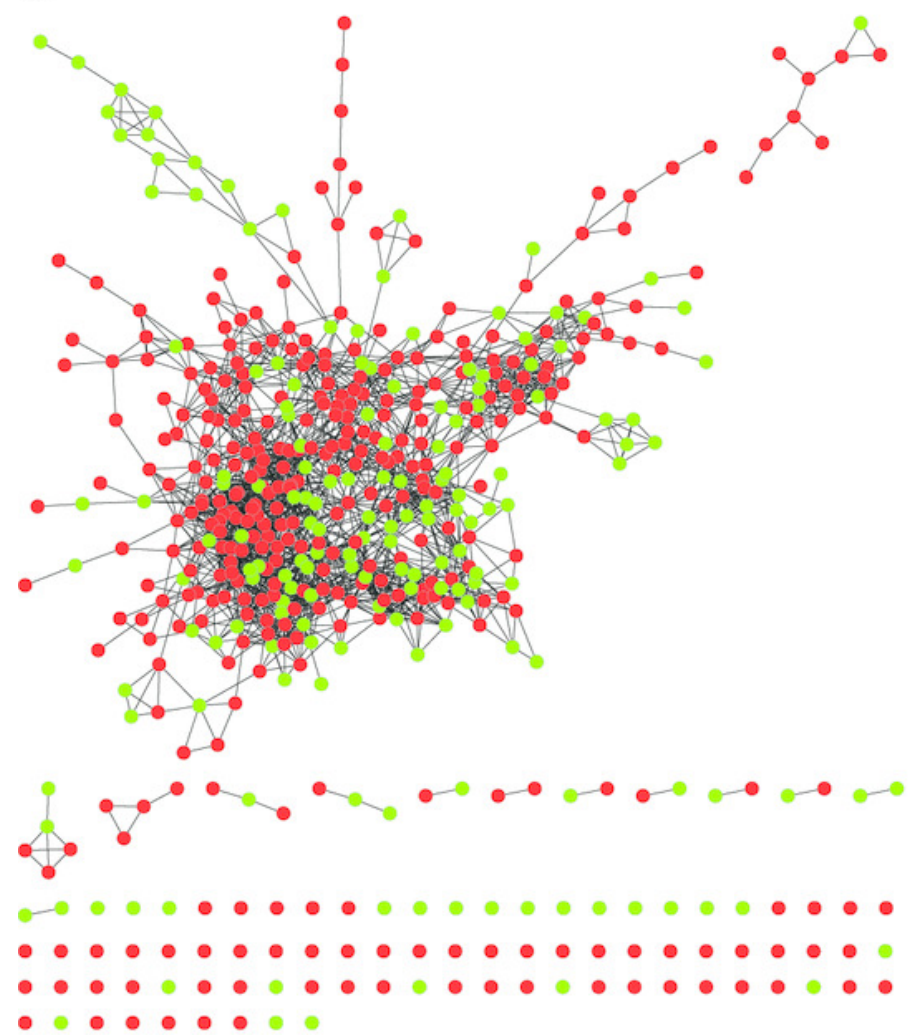

Average number of neighbors $=9.381$ (2500 edges)

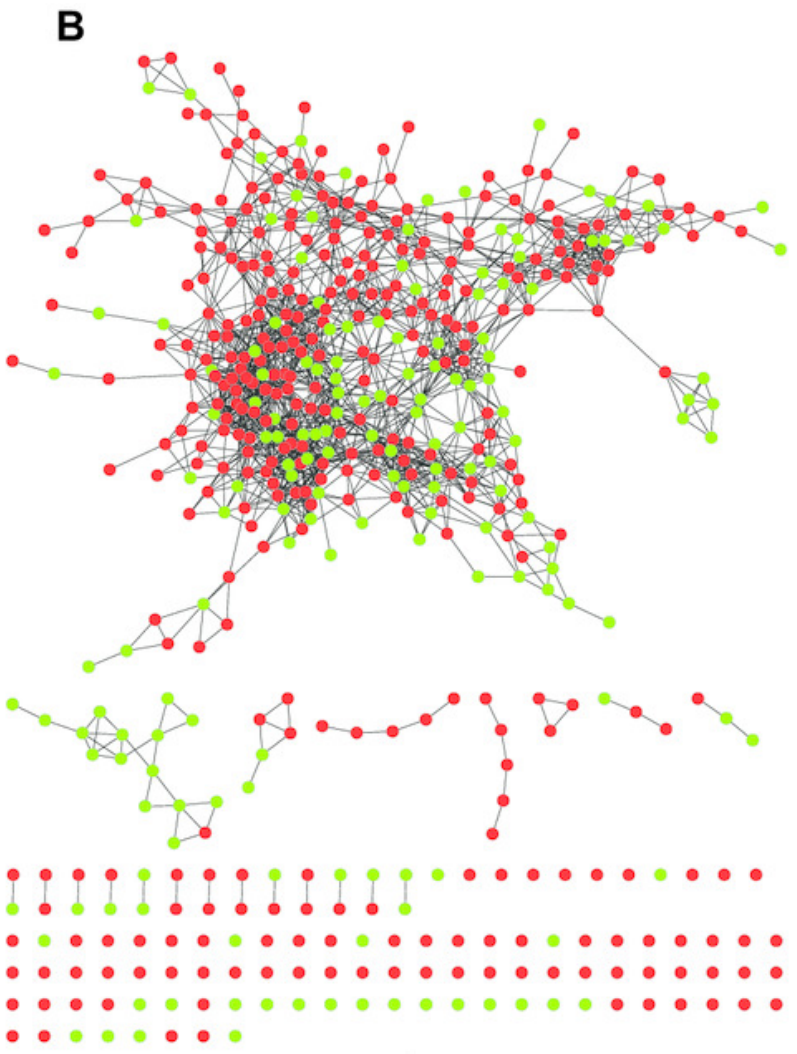

Average number of neighbors $=7.505$ (2000 edges)

D

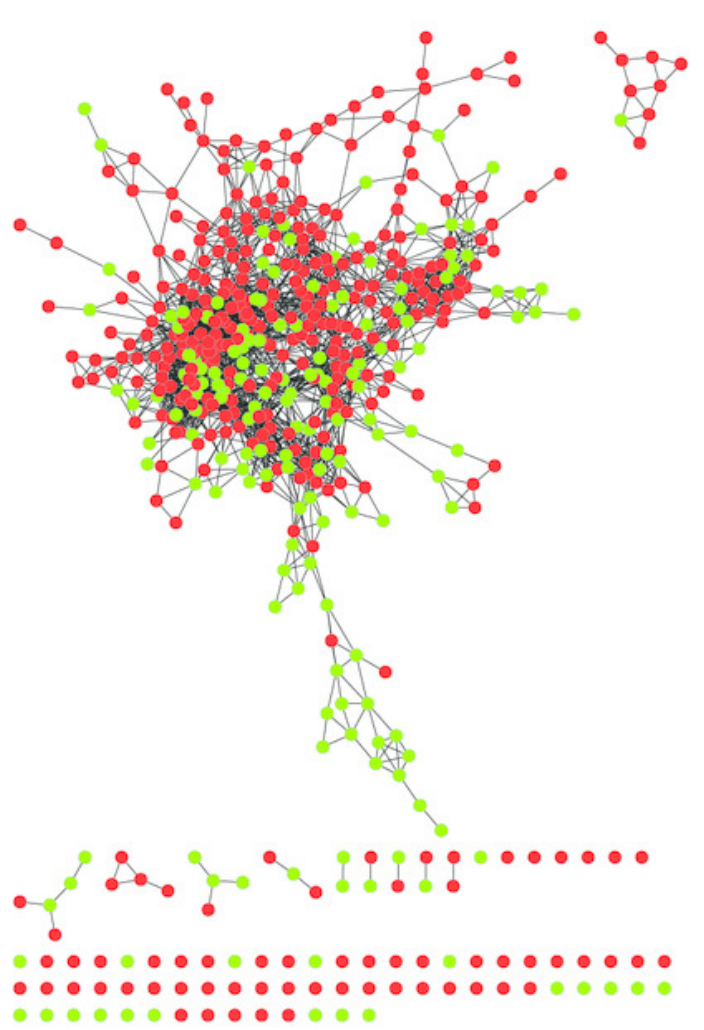

Average number of neighbors $=11.257$ (3000 edges) 


\section{Figure 3}

Figure 3. Optimization and evaluation of ANN II.

A. Distribution of the Youden index values of ANN models built by dividing all the patients with healthy lungs and those complicated with ILD into 5, 10, 15, or 20 groups, respectively. The first ANN models of different division sizes 5D1, 10D1, 15D1, and 20D1 are highlighted as larger dots. The position of the dotted line is 0.387 , and values of Youden index were calculated using ANN I. B. Comparison of ANNs I and II in ROC curves. A significant difference in AUCs was found $(P=0.025)$. C. The 10 -fold cross-validation (10FCV) result of ANN II.

A

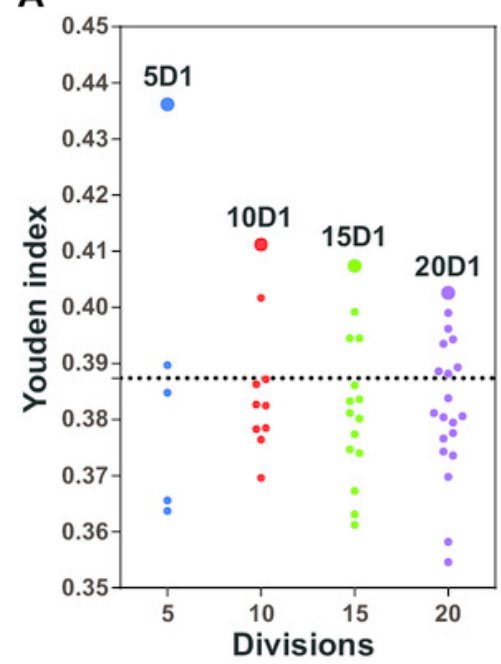

B

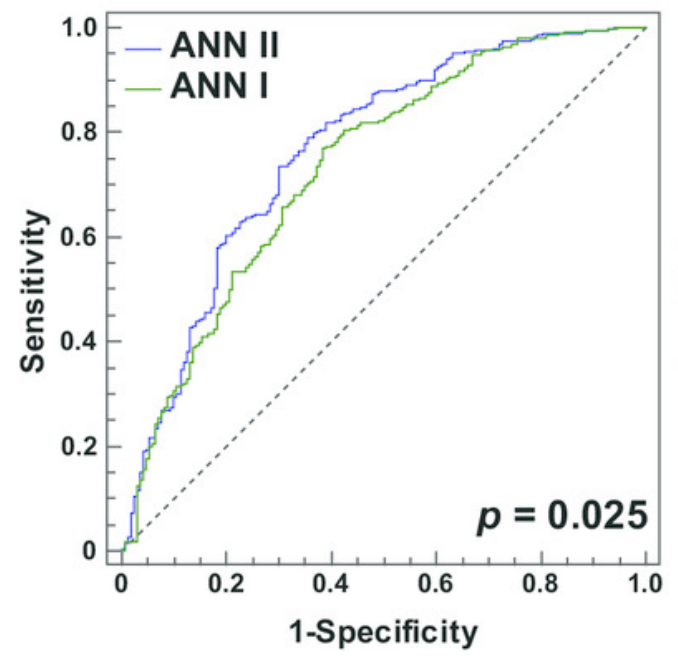

C

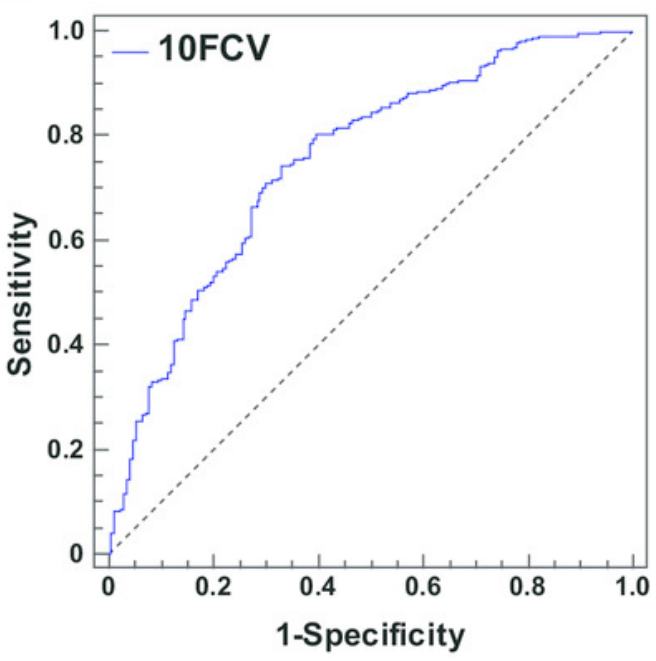


Figure 4

Figure 4. Model evaluation using an independent patient set of 87 RA patients with PF and an external validation set of 218 RA patients.

A. Comparison of single ILD-associated CEls, ANNs I and II in identifying patients with PF. B. Scatter plots of ANN II outputs for patients with healthy lungs and those complicated with ILD

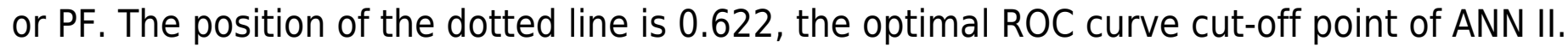

C. The external validation result of ANN II.

A

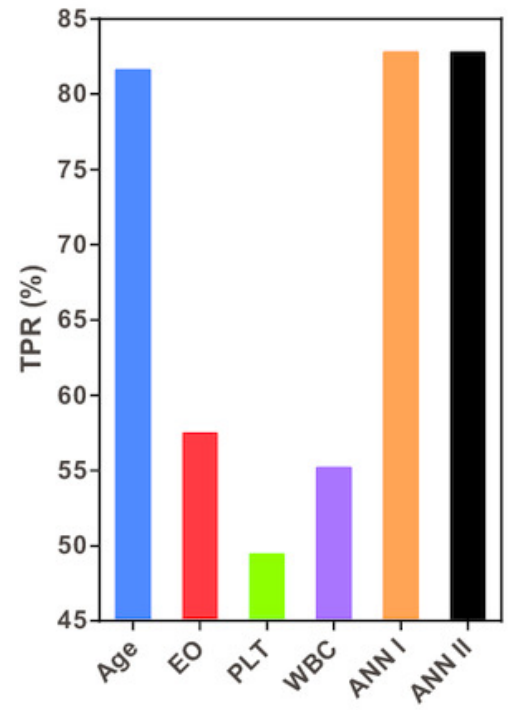

B

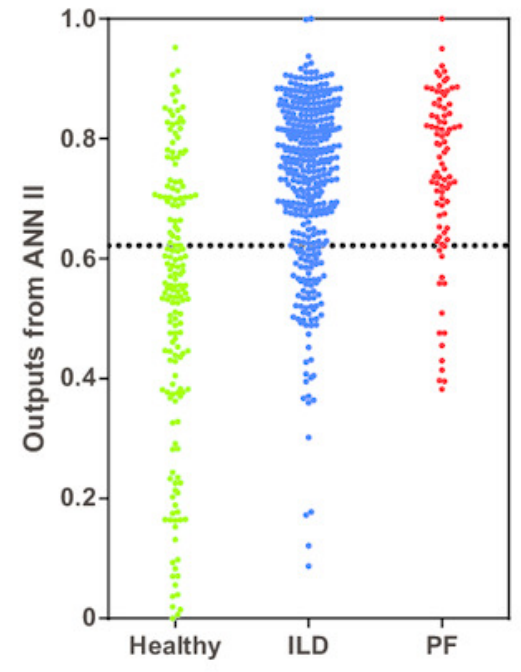

C

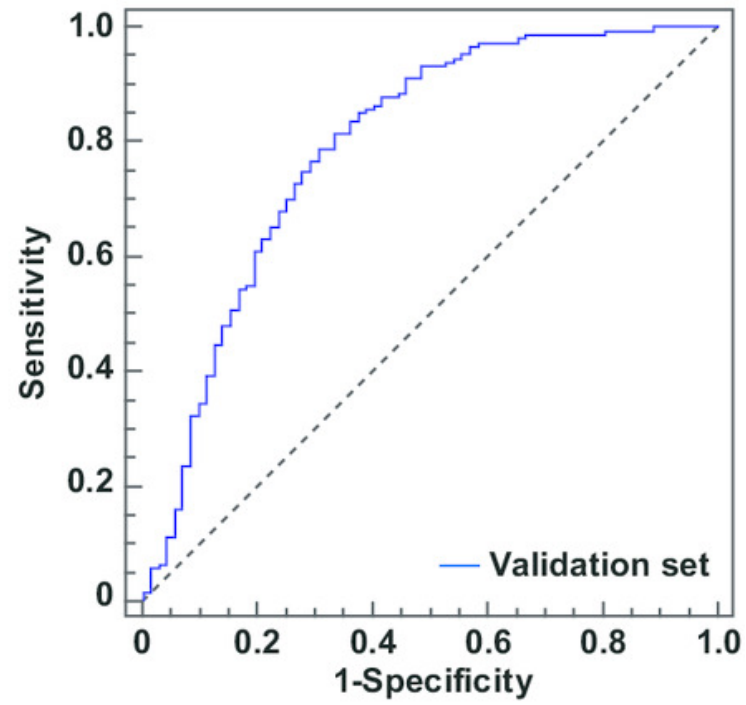

